\title{
A new PQCD based Monte Carlo event generator for jets in the quark-gluon plasma
}

\author{
Paul Caucal* \\ Institut de Physique Théorique, Université Paris-Saclay, CNRS, CEA, F-91191, Gif-sur-Yvette, \\ France \\ E-mail: paul.caucaldipht.fr

\section{Edmond lancu} \\ Institut de Physique Théorique, Université Paris-Saclay, CNRS, CEA, F-91191, Gif-sur-Yvette, \\ France \\ E-mail: edmond.iancueipht.fr
}

\author{
Alfred H. Mueller \\ Department of Physics, Columbia University, New York, NY 10027, USA \\ E-mail: amh@phys.columbia.edu
}

\section{Gregory Soyez}

Institut de Physique Théorique, Université Paris-Saclay, CNRS, CEA, F-91191, Gif-sur-Yvette, France

E-mail: gregory.soyez@ipht.fr

\begin{abstract}
A main difficulty in understanding the dynamics of jets produced in the high-density environment of ultrarelativistic heavy ion collision, is to provide a unified description for the two sources of radiation that are a priori expected: the "vacuum-like" emissions responsible for the parton shower from large virtualities down to the hadronisation scale and the "medium-induced" emissions responsible for the energy loss by the jet. In a recent paper [1], we demonstrated that these two mechanisms can be factorized from each other within a controlled, "double-logarithmic" approximation in perturbative QCD. In this proceeding we recall the main features of the jet evolution in a dense QCD medium. We emphasize that the in-medium parton showers differ from those in the vacuum in two crucial aspects: their phase-space is reduced and the first emission outside the medium can violate angular ordering. Based on this factorized picture, which is Markovian, we have recently developed a Monte Carlo event generator which includes both vacuum-like and medium-induced emissions and goes beyond the double logarithmic approximation. We here present our first results for the fragmentation function and the energy loss by the jet.
\end{abstract}

International Conference on Hard and Electromagnetic Probes of High-Energy Nuclear Collisions 30 September - 5 October 2018

Aix-Les-Bains, Savoie, France

${ }^{*}$ Speaker. 


\section{Introduction}

One important goal of the experimental programs at RHIC and at the LHC is the characterisation of the quark-gluon plasma (QGP) produced in ultrarelativistic heavy ion collisions. Jet quenching observables, i.e. the modifications of the properties of an energetic jet due to its interactions with the dense form of QCD matter produced right after a collision, are particularly interesting to study the properties of this deconfined phase.

The suppression of the inclusive hadron and jet cross-sections in nucleus-nucleus collision w.r.t proton-proton was the first historical indication of jet energy loss in the plasma and has been accurately measured at both RHIC and the LHC [2, 3, 4, 5]. Most recently, substructure observables such as the jet fragmentation function $[6,7]$ have provided new insights on the evolution of a jet in the QGP.

From a theoretical point of view, high- $p_{T}$ jets are particularly advantageous because one can rely on perturbative $\mathrm{QCD}$ (pQCD) to understand their properties. Within this approach, the dense weakly-coupled quark-gluon medium may trigger medium-induced radiations from the virtual partons inside jets. This can be computed with the BDMPS-Z formalism [8, 9, 10], recently extended to include multiple branchings $[11,12,13,14,15]$. Nevertheless, the global jet structure has to be built with the usual, "vacuum-like" bremsstrahlung process through which a parton loses its virtuality from the hard scale until the hadronisation.

In a recent paper [1], a landmark has been achieved in the understanding of the interplay between these two mechanisms, vacuum-like emissions (VLEs) and medium induced radiations. We showed that the double-logarithmic approximation (DLA) of pQCD provides a rigorous framework to deal with the jet evolution in a QGP and should be regarded as a first step toward a more advanced treatment including other physical effects.

In this proceeding, we first recall the crucial aspects of double-logarithmic parton cascades in the QGP. Then, we explain how the probabilistic picture that emerges from the DLA has been implemented in a new Monte Carlo (MC) event generator which accounts for both vacuum-like emissions and medium induced radiations. We finally present preliminary results for the nuclear modification factor and the jet fragmentation function.

\section{Jet evolution in the presence of a medium at DLA}

After having recalled the basic properties of jet evolution in the vacuum, this section sums up how the medium changes the development of parton cascades.

\subsection{Evolution in the vacuum}

In the vacuum, the two building blocks to construct a parton shower are the Bremsstrahlung process which provides the probability law for a soft and collinear emission by a virtual parton and the angular ordering property which enables one to treat successive Bremsstrahlung emissions as a Markov chain of independent elementary radiation events, ordered in angles [16].

The specific feature of the Bremsstrahlung probability distribution $d^{2} \mathscr{P}_{\mathscr{B}}$ is the logarithmic enhancement of soft and collinear emissions:

$$
d^{2} \mathscr{P}_{\mathscr{B}}=\frac{\alpha_{s} C_{R}}{\pi} \frac{d \omega}{\omega} \frac{d \theta^{2}}{\theta^{2}}
$$


The essence of the DLA is the resummation of contributions coming from such processes to all orders in the computation of intrajet observables.

\subsection{Factorization between VLEs and medium induced radiations}

In addition to the vacuum-like emissions, the dense weakly-coupled medium triggers radiations à la BDMPS-Z if the formation time of the medium-induced parton is much larger than the mean free path between successive collisions off the medium constituents. Thereafter, our QGP is characterized by only two parameters: the distance $L$ covered by the jet inside the medium and the jet quenching parameter $\hat{q}$ corresponding to the averaged transverse momentum acquired by multiple collisions during a time $\Delta t$ via the relation $\left\langle k_{\perp}^{2}\right\rangle=\hat{q} \Delta t$.

In this framework, the probability distribution $d \mathscr{P}_{\text {med }}$ for medium induced processes is well approximated by the formula $[17,18]$

$$
d \mathscr{P}_{\mathrm{med}} \simeq \frac{\alpha_{s} N_{c}}{\pi} L \sqrt{\frac{\hat{q}}{\omega^{3}}} d \omega
$$

This spectrum shows no collinear nor soft logarithmic enhancement so one can safely ignore the medium-induced radiations when computing the intrajet multiplicity at DLA.

Even if at DLA, the medium seems to have no direct effects, it will appear as a new constraint in the phase space for VLEs and this constraint will lead to the factorization between the vacuumlike showers and the medium induced effects. Indeed, a VLE occurring inside a medium has a minimal transverse momemtum $k_{\perp}^{\min }=\sqrt{\hat{q} t_{f}}$ given by the momentum acquired via multiple collisions during its formation time $t_{f}$. This minimal transverse momentum condition, once translated in terms of the angle and energy of emission through the uncertainty principle relation $t_{f} \simeq 1 / \omega \theta^{2}$ becomes a lower bound in the phase space represented by the line $\omega^{3} \theta^{4}=2 \hat{q}$ in Fig. 1 (left). Of course, since the medium has a finite length, a VLE can also take place directly outside the medium if its formation time $t_{f}$ is larger than $L$. Again, this constraint on VLE outside the medium translates into an upper bound in the energy/angle phase space represented by the line $\omega \theta^{2} L=2$ in Fig. 1 (left).

As an immediate consequence of the previous discussion, the phase space for VLEs is reduced by the presence of a vetoed region where there is no VLE permitted as shown in Fig. 1 (left).

As already outlined, another important consequence is the possibility to factorize VLEs from medium induced emissions: the latter are neglected at DLA but we would eventually like to include them to account for energy loss and also to compute the jet fragmentation beyond DLA. The probabilistic picture for the jet evolution that emerges from the DLA analysis is the following: firstly, a vacuum-like shower develops itself during a very short time $\ll L$ in the in-medium region ("inside" region, Fig. 1 (left)). Then the partons that come out of this shower undergo medium-induced processes. Especially, they become seeds for medium-induced mini-jets as studied in references $[12,13,14,15,19]$. Then, when the partons come out of the medium (they are in the "outside" region, Fig. 1 (left)), a usual angular ordered vacuum shower decreases their virtualities down to the hadronisation scale $\Lambda_{\mathrm{QCD}}$. 

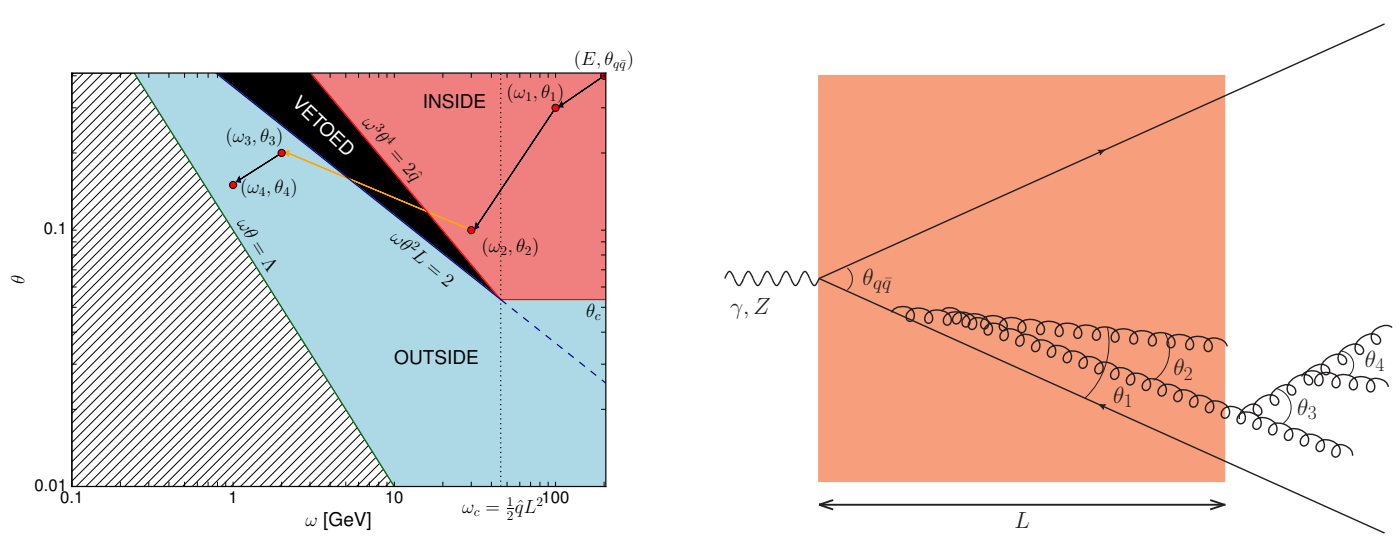

Figure 1: (Left) Phase space for one VLE in a jet of energy $E=200 \mathrm{GeV}$ and opening angle $\bar{\theta}=0.4$ in the presence of a medium. Above the red line, $k_{\perp} \geq k_{\perp}^{\min }=\sqrt{\hat{q} t_{f}}$ and below the blue line $t_{f} \geq L$. In the hatched region $k_{\perp} \leq \Lambda_{\mathrm{QCD}}$, partons have hadronised and the pQCD regime is not valid anymore. (Right) Cascade which might happen in the presence of a medium but not in the vacuum. The position of corresponding successive emissions in the phase space are represented on the left. Angular ordering is violated by the third emission, but preserved by all the others. The yellow arrow between the second and the third emission represents all the medium induced processes that can affect the parton 2 traveling through the medium over a large distance.

\subsection{Violation of angular ordering}

Medium induced radiation is not the only effect of the QGP that matters for the jet evolution. When previously discussing parton showers in the vacuum, we outlined the importance of the color coherence and angular ordering to describe a jet as a branching process.

A color singlet dipole with opening angle $\bar{\theta}$ propagating through the medium may lose its color coherence via multiple scattering off the medium: the two prongs suffer independent color rotations, hence the probability that the dipole remains in a color singlet state decreases with time $[20,21,22]$. The two legs of the antenna start behaving like independent color sources after a time $t_{c o h}(\bar{\theta}) \equiv\left(4 / \hat{q} \bar{\theta}^{2}\right)^{1 / 3}[21]$. Consequently, angular ordering could be violated in the presence of a medium.

Yet, in Ref [1], we argue that this is not the case for VLEs occurring inside the medium: the respective formation times are small enough for the effect of color decoherence to be negligible. Hence, to DLA at least, angular ordered cascades develop inside the medium exactly as in the vacuum, as drawn in Fig. 1 (right).

However, the last antenna produced inside the medium by the in-medium vacuum-like shower plays a special role. Indeed, this antenna will have to travel through the medium over a distance of $\operatorname{order} L$. Calling $\theta_{\text {last }}$ its opening angle, two cases are possible: either $t_{\text {coh }}\left(\theta_{\text {last }}\right) \leq L$ or $t_{\text {coh }}\left(\theta_{\text {last }}\right)>$ $L$. In the first case which corresponds to $\theta_{\text {last }} \geq \theta_{c} \equiv 2 / \sqrt{\hat{q} L^{3}}$ the last antenna hast lost its color coherence so the next emission outside the medium can radiate at any angle. In the second case, the antenna is still coherent at the time of formation of the next one. Consequently, the angle of the next emission is constrained by the angle $\theta_{\text {last }}$. The introduction of the critical angle $\theta_{c}$ leads to a modification of the "inside" region in Fig. 1 (left): this region is now defined by $k_{\perp} \geq \sqrt{\hat{q} t_{f}}$ and 
$\theta \geq \theta_{c}$. In this region, cascades are always angular ordered.

To summarize, the DLA analysis of jet evolution in a dense QCD medium has exhibited three important features: a vetoed region for VLEs, the factorization of medium-induced processes between the angular ordered in-medium and out-medium showers and finally one violation of angular ordering by the first emission outside the medium is permitted $[1,23]$.

\section{Monte Carlo event generator}

At this stage of the discussion, it should be clear that the probabilistic picture sketched in the previous section is particularly suitable for a MC implementation which presents also the advantage to take easily into account the conservation of energy and the running of the strong coupling constant through the evolution.

\subsection{Brief description of the Monte Carlo implementation}

The MC event generator uses two modules: the first one deals with the vacuum-like angularordered shower (inside or outside the medium) and the second one implements the medium-induced cascades for which the branching rate is given by the BDMPS- $\mathrm{Z}$ rate following the ideas developed in Refs $[12,13]$.

\subsubsection{Vacuum-like shower}

In order to generalize the results obtained in Ref [1] for the fragmentation function at DLA, the vacuum-like shower implements the energy conservation and DGLAP splitting probabilities at every branching. The color representations of the partons (either quark $q$ or gluons $g$ ) are properly treated.

However at this level of accuracy, the angular ordering property is valid only after averaging over the azimuthal angle of emission. To be consistent with this principle from $\mathrm{pQCD}$, an azimuthal angle is chosen randomly between 0 and $2 \pi$ at each emission.

Because of angular ordering, the evolution parameter of the branching process is naturally the angle of a parton with respect to its emitter. In the vacuum, the Sudakov form factor $\Delta_{i}\left(\bar{\theta}^{2}, \theta_{0}^{2}\right)$ is the probability to have no branching between the angle $\bar{\theta}$ of the parent parton of type $i \in\{q, g\}$ with energy $E$ and a smaller angle $\theta_{0}$ :

$$
\Delta_{i}\left(\bar{\theta}^{2}, \theta_{0}^{2}\right)=\exp \left(-\int_{\theta_{0}^{2}}^{\bar{\theta}^{2}} \frac{d \theta^{2}}{\theta^{2}} \int_{0}^{1} d z \frac{\alpha_{s}(z E \theta)}{2 \pi} P_{j i}(z) \Theta\left(z E \theta>k_{\perp}^{c}\right)\right)
$$

where $P_{i j}(z)$ are the leading order DGLAG splitting functions and $\alpha_{s}(z E \theta)$ is the one-loop QCD running coupling evaluated at the transverse momentum of the emitted parton.

The step function $\Theta\left(z E \theta>k_{\perp}^{c}\right)$ enforces the condition that the $k_{\perp} \simeq z E \theta$ of the emission has to be larger than a given cut-off $k_{\perp}^{c}$. Inside the medium, this $k_{\perp}^{c}$ corresponds to the boundary of the "inside" region in Fig. 1 (left) while outside the medium, this is a scale of order $\Lambda_{\mathrm{QCD}}$. In practice, a Sudakov veto algorithm based on Equ. 3.1 is used to produce a branching angle.

In Fig. 2 (left), we show our MC results for the fragmentation function as produced by VLEs alone. Accordingly, the only effect of the medium is to introduce the vetoed region and the reopening of the phase space for the first emission outside. For comparison, we also show our MC 
predictions for jets propagating in the vacuum as well as the medium/vacuum ratio. The enhancement visible in this ratio at low $p_{T}$ is a consequence of decoherence whereas the depletion at intermediate energy is a consequence of the veto. There is also an enhancement at large $p_{T}$ due to the fact that, in the presence of the vetoed region, the leading particle has less probability to radiate and hence a larger probability to carry a large value of $z$.
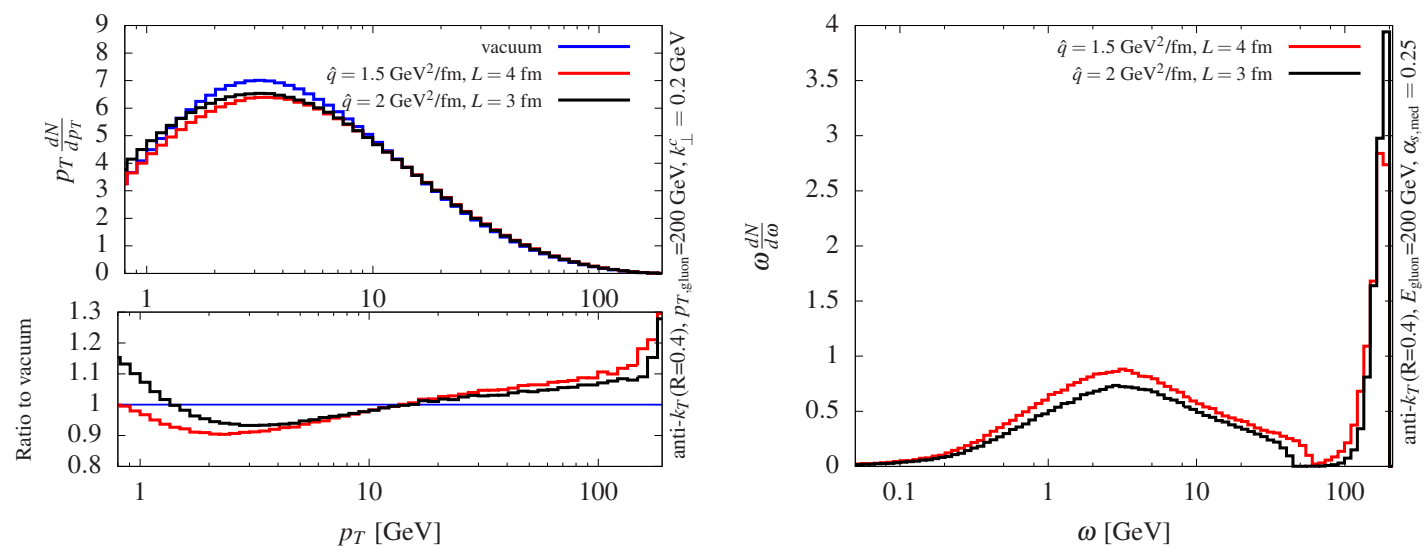

Figure 2: (Left) In blue, the fragmentation function for a jet in the vacuum created by a gluon with $p_{T, \text { gluon }}=$ $200 \mathrm{GeV}$ as given by the module "vacuum-like shower" of our MC event generator. The other curves correspond to the fragmentation function in the presence of a medium with only VLEs for two sets of medium parameters. Below, the ratios with respect to the vacuum are plotted. (Right) The energy spectrum of particles produced by medium-induced splittings from a leading gluon with $E_{\text {gluon }}=200 \mathrm{GeV}$ resulting from the module "medium-induced shower", for two different values of $\hat{q}$ and $L$. The parameters $z_{c}$ and $\theta_{\max }$ are fixed respectively to $10^{-5}$ and 1 .

\subsubsection{Medium-induced parton shower}

This module develops a medium-induced parton shower from a leading parton with energy $E$. The evolution parameter in the case of medium induced cascades is the "time" $t=x^{+}$in light-cone coordinates with the longitudinal axis defined by the direction of motion of the leading particle [12]. The branching rate associated with the splitting of a gluon with energy fraction $x=\omega / E$ into two gluons, one carrying away a fraction $z$ of the energy is [12]

$$
\frac{d^{2} \mathscr{P}_{b r}}{d z d t}=\frac{1}{2 \pi} \frac{P_{g g}(z)}{\tau_{b r}(z, x)}, \tau_{b r}(z)=\frac{1}{\alpha_{s}} \sqrt{\frac{z(1-z) x E}{\hat{q}_{\text {eff }}(z)}}, \hat{q}_{\text {eff }}(z)=\hat{q}(1-z(1-z))
$$

A similar formula exists for a quark splitting and is incorporated as well in our code. In the mediuminduced parton shower, the strong coupling constant is assumed to take a fixed value $\alpha_{s, \text { med }}$. The treatment of the running of the coupling is left for further studies.

To produce a branching, one needs first to compute the Sudakov form factor associated with this branching rate in order to generate the next branching time. The probability $\Delta_{\text {med }}\left(\bar{t}, t_{0}\right)$ to have no branching betweem times $\bar{t}$ and $t_{0}$ is

$$
\Delta_{\text {med }}\left(\bar{t}, t_{0}\right)=\exp \left(-\int_{\bar{t}}^{t_{0}} d t \int_{z_{c} / x}^{1-z_{c} / x} d z \frac{d^{2} \mathscr{P}_{b r}}{d z d t}\right)
$$


with a cut-off $z_{c}$ in the energy fraction.

Here again, a Sudakov veto algorithm is used to pick the subsequent branching time. Then, we generate a given energy fraction $z$ according to the effective splitting probability $P_{g g}(z) / \tau_{b r}(z, x)$.

At this stage, every splitting is assumed to be collinear with the leading particle. Indeed, as well known most of the transverse momemtum broadening is acquired during the propagation time $\Delta t$ between two successive branchings. In our MC, this is mimicked by ascribing to each parton an average transverse momentum $\left\langle k_{\perp}^{2}\right\rangle=\hat{q} \Delta t$ with a Gaussian distribution. Evolution is stopped if the branching time is larger than the length of the medium $L$ or if the momentum broadening has pushed the angle of emission beyond a maximal angle $\theta_{\max } \simeq 1$ or finally if the energy fraction with respect to the leading particle carried by the parton is smaller than $z_{c}$.

In Fig. 2 (right), we show the resulting energy spectrum of the medium-induced shower from an initial gluon with $E=200 \mathrm{GeV}$. The large narrow peak near $\omega=200 \mathrm{GeV}$ is the remnant of the leading particle. The second broad peak corresponds to the accumulation around the characteristic transverse momemtum scale $Q_{s}=\sqrt{\hat{q} L}$ of soft gluons produced via medium induced radiations due to momentum broadening. This spectrum agrees with previous studies [12, 13, 15, 19].
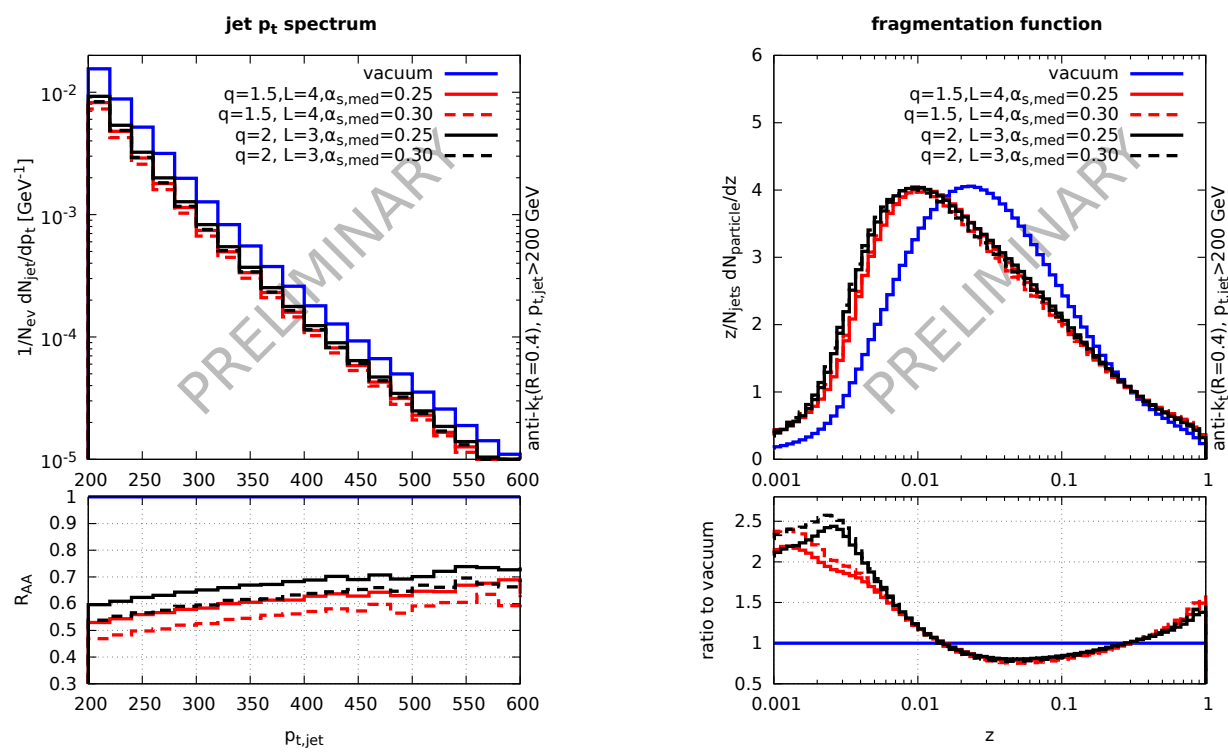

Figure 3: (Left) Jet $p_{T}$ spectrum in the vacuum and in the presence of a medium from our MC event generator for several values of $\hat{q}$ and $L$ and nuclear modification factor for jets $R_{A A}^{\text {jet }}$. (Right) Fragmentation function at the partonic level. All jets with $p_{T, \text { jet }} \geq 200 \mathrm{GeV}$ have been selected for these analysis.

\subsection{Results and discussion}

In this section, some preliminary results of the full MC are shortly discussed. The hard process is generated from a list of hard events in di-jet production at LHC with $\sqrt{s}=5.02 \mathrm{TeV}$. Then, the two partons created by this hard process are showered until the border of the in-medium region with the vacuum-like shower module. All the partons created by this first cascade become seeds of medium-induced cascades handled by the medium-induced parton shower. Finally, the resulting particles of the previous shower are evolved in the "outside" region until hadronisation with the 
vacuum shower module. To account for color decoherence, all the partons produced inside the medium (via either vacuum-like or medium induced emissions) are allowed to radiate at any angle.

For the jet reconstruction in the final state, the sofware package FastJet is used [24]. Jets are reconstructed by the anti- $k_{T}$ algorithm [25] with $R=0.4$.

In Fig. 3, we show the results for the nuclear modification factor for jets $R_{A A}^{\text {jet }}$ and the ratio $R_{D(z)}$ of the fragmentation function in nucleus-nucleus collisions and proton-proton collisions. Concerning the $R_{A A}^{\text {jet }}$ factor, our MC predicts very well the flatness of the data [4, 5] at high $p_{T}$. Physically, this is a consequence of the fact that energy loss is stronger for a jet than for a single parton and is increasing with the jet energy (due to enhanced radiations).

The ratio $R_{D(z)}$ shows the same qualitative behaviour as LHC data [6, 7] notably an enhancement at both very small and relatively large values of $z$, and a depletion in between. In our picture the enhancement at low $z$ is due to the violation of angular ordering by the first emission outside the medium. The enhancement at large $z$ comes from the fact that a single hard parton inside the jet loses less energy than the jet as a whole.

\section{Conclusion}

In this proceeding, we briefly discussed a new picture, emerging from $\mathrm{pQCD}$ for the evolution of a jet propagating through a dense QCD plasma. We have furthermore presented the first results of a Monte Carlo event generator which is grounded in this picture. Our preliminary results favorably compare to the data. In the future, we plan to perform more systematic studies of phenomenology and also to extend our theoretical framework by including missing physical ingredients such as hadronisation and a more realistic geometry of the medium.

A more thorough justification of the validity of our picture beyond DLA together with more details on the MC implementation will be presented in an upcoming publication.

Acknowledgements. The work of P.C., E.I. and G.S. is supported in part by the Agence Nationale de la Recherche project ANR-16-CE31-0019-01. The work of A.H.M. is supported in part by the U.S. Department of Energy Grant \# DE-FG02-92ER40699.

\section{References}

[1] P. Caucal, E. Iancu, A. H. Mueller and G. Soyez, Vacuum-like jet fragmentation in a dense QCD medium, Phys. Rev. Lett. 120 (2018) 232001 [1801.09703].

[2] STAR collaboration, C. Adler et al., Centrality dependence of high $p_{T}$ hadron suppression in $A u+A u$ collisions at $\sqrt{s}_{N N}=130-G e V$, Phys. Rev. Lett. 89 (2002) 202301 [nucl-ex/0206011].

[3] PHENIX collaboration, K. Adcox et al., Suppression of hadrons with large transverse momentum in central Au+Au collisions at $\sqrt{s_{N N}}=130-G e V$, Phys. Rev. Lett. 88 (2002) 022301 [nucl-ex/0109003].

[4] CMS collaboration, V. Khachatryan et al., Measurement of inclusive jet cross sections in pp and PbPb collisions at $\sqrt{s_{N N}}=2.76 \mathrm{TeV}$, Phys. Rev. C96 (2017) 015202 [1609. 05383].

[5] ATLAS collaboration, M. Aaboud et al., Measurement of the nuclear modification factor for inclusive jets in $P b+P b$ collisions at $\sqrt{s_{\mathrm{NN}}}=5.02 \mathrm{TeV}$ with the ATLAS detector, 1805.05635. 
[6] CMS collaboration, S. Chatrchyan et al., Measurement of jet fragmentation in PbPb and pp collisions at $\sqrt{s_{N N}}=2.76 \mathrm{TeV}$, Phys. Rev. C90 (2014) 024908 [1 406.0932 ].

[7] ATLAS collaboration, M. Aaboud et al., Measurement of jet fragmentation in $\mathrm{Pb}+\mathrm{Pb}$ and $\mathrm{pp}$ collisions at $\sqrt{s_{N N}}=5.02 \mathrm{TeV}$ with the ATLAS detector, Phys. Rev. C98 (2018) 024908 [1805.05424].

[8] R. Baier, Y. L. Dokshitzer, A. H. Mueller, S. Peigne and D. Schiff, Radiative energy loss of high-energy quarks and gluons in a finite volume quark - gluon plasma, Nucl. Phys. B483 (1997) 291 [hep-ph/9607355].

[9] B. G. Zakharov, Fully quantum treatment of the Landau-Pomeranchuk-Migdal effect in QED and QCD, JETP Lett. 63 (1996) 952 [hep-ph/9607440].

[10] U. A. Wiedemann, Gluon radiation off hard quarks in a nuclear environment: Opacity expansion, Nucl. Phys. B588 (2000) 303 [hep-ph/ 0005129 ].

[11] J.-P. Blaizot, F. Dominguez, E. Iancu and Y. Mehtar-Tani, Medium-induced gluon branching, JHEP 01 (2013) 143 [1209.4585].

[12] J.-P. Blaizot, E. Iancu and Y. Mehtar-Tani, Medium-induced QCD cascade: democratic branching and wave turbulence, Phys. Rev. Lett. 111 (2013) 052001 [1301.6102].

[13] J.-P. Blaizot, Y. Mehtar-Tani and M. A. C. Torres, Angular structure of the in-medium QCD cascade, Phys. Rev. Lett. 114 (2015) 222002 [1407.0326].

[14] A. Kurkela and U. A. Wiedemann, Picturing perturbative parton cascades in QCD matter, Phys. Lett. B740 (2015) 172 [1407.0293].

[15] M. A. Escobedo and E. Iancu, Event-by-event fluctuations in the medium-induced jet evolution, JHEP 05 (2016) 008 [1601.03629].

[16] Y. L. Dokshitzer, V. A. Khoze, A. H. Mueller and S. I. Troian, Basics of perturbative QCD. 1991.

[17] C. A. Salgado and U. A. Wiedemann, Calculating quenching weights, Phys. Rev. D68 (2003) 014008 [hep-ph/0302184].

[18] U. A. Wiedemann, Jet quenching versus jet enhancement: A Quantitative study of the BDMPS-Z gluon radiation spectrum, Nucl. Phys. A690 (2001) 731 [hep-ph/0008241].

[19] L. Fister and E. Iancu, Medium-induced jet evolution: wave turbulence and energy loss, Journal of High Energy Physics 2015 (2015) 82.

[20] Y. Mehtar-Tani, C. A. Salgado and K. Tywoniuk, Anti-angular ordering of gluon radiation in QCD media, Phys. Rev. Lett. 106 (2011) 122002 [1009.2965].

[21] J. Casalderrey-Solana and E. Iancu, Interference effects in medium-induced gluon radiation, JHEP 08 (2011) 015 [1105.1760].

[22] J. Casalderrey-Solana, Y. Mehtar-Tani, C. A. Salgado and K. Tywoniuk, New picture of jet quenching dictated by color coherence, Phys. Lett. B725 (2013) 357 [1210 . 7765].

[23] Y. Mehtar-Tani and K. Tywoniuk, Jet (de)coherence in PbPb collisions at the LHC, Phys. Lett. B744 (2015) 284 [1401.8293].

[24] M. Cacciari, G. P. Salam and G. Soyez, FastJet User Manual, Eur. Phys. J. C72 (2012) 1896 [1111.6097].

[25] M. Cacciari, G. P. Salam and G. Soyez, The anti-k $k_{t}$ jet clustering algorithm, JHEP 04 (2008) 063 [0802.1189]. 